\title{
COVID-19 clinical risk assessment for guiding patient placement and diagnostic testing strategy on admission to hospital
}

\author{
Nick K Jones ${ }^{1}$, Isobel Ramsay ${ }^{1}$, Elinor Moore ${ }^{1}$, Jonathan Fuld ${ }^{1}$, Chris Adcock ${ }^{1}$, Edward \\ Banham-Hall ${ }^{1}$, Judith Babar ${ }^{1}$, Effrossyni Gkrania-Klotsas ${ }^{1}$, and Hoi Ping Mok ${ }^{1}$ \\ ${ }^{1}$ Affiliation not available
}

October 21, 2020

\begin{abstract}
Introduction: Without universal access to point-of-care SARS-CoV-2 testing, many hospitals rely on clinical judgement alone for identifying cases of COVID-19 early.

Methods: Cambridge University Hospitals NHS Foundation Trust introduced a traffic lights clinical judgement aid to the COVID-19 admissions unit in mid-March 2020. Ability to accurately predict COVID-19 was audited retrospectively across different stages of the epidemic.

Results: One SARS-CoV-2 PCR positive patient (1/41, 2\%) was misallocated to a 'green' (non-COVID-19) area during the first period of observation, and no patients $(0 / 32,0 \%)$ were mislabelled 'green' during the second period. 33 of 62 (53\%) labelled 'red' (high risk) tested SARS-CoV-2 PCR positive during the first period, while 5 of 22 (23\%) 'red' patients were PCR positive in the second.

Conclusion: COVID-19 clinical risk stratification on initial assessment effectively identifies non-COVID-19 patients. However, diagnosing COVID-19 is challenging and risk of overcalling COVID-19 should be recognised, especially when background incidence is low.
\end{abstract}

Key words: SARS-CoV-2, COVID-19, Coronavirus, Triage, Diagnostics, Infection Control

\section{Hosted file}

Title page.docx available at https://authorea.com/users/332647/articles/488112-covid-19clinical-risk-assessment-for-guiding-patient-placement-and-diagnostic-testing-strategyon-admission-to-hospital

Admission COVID-19 clinical risk assessment for guiding patient placement and diagnostic testing strategy

The COVID-19 pandemic is creating enormous logistical challenges for health services across the UK, with many hospitals having been forced to restructure systems that have been in place for decades. Major priorities in the re-design of pathways for patient admission are to ensure potentially infectious patients are kept separate from those that remain susceptible, and to utilise testing capacity rationally and effectively. Until point-of-care (POC) SARS-CoV-2 testing becomes universally available ${ }^{1}$, clinical judgement will continue to form the basis of patient placement decisions. Here, we report our experience of using a clinical risk stratification system developed in our hospital.

Similar to other hospitals, ${ }^{2}$ we have been using a COVID-19 risk stratification system to categorise patients according to how readily their presenting symptoms, clinical signs, POC blood test results and chest x-ray images can be explained by COVID-19 or alternative diagnoses (Table 1). Traffic light colours are assigned 
to individual cases based on clinical judgement during initial assessment at the time of admission. Such clinical risk assessment requires prior knowledge of the typical presenting features of COVID-19, but does not involve the use of strict diagnostic criteria and is not a validated diagnostic or prognostic tool. It invites the physician to consider the extent to which the presenting clinical features can be explained by COVID-19 or an alternative diagnosis. We introduced the system in combination with a succinct summary table of the most commonly reported clinical, POC laboratory and radiological findings in COVID-19 cases (Table 2), to aid non-specialist clinicians in the assessment of this novel disease. Illustrative examples of cases assigned to various cohorts are described in box 1.

\section{Hosted file}

Table 1.docx available at https://authorea.com/users/332647/articles/488112-covid-19clinical-risk-assessment-for-guiding-patient-placement-and-diagnostic-testing-strategyon-admission-to-hospital

\section{Hosted file}

Table 2.docx available at https://authorea.com/users/332647/articles/488112-covid-19clinical-risk-assessment-for-guiding-patient-placement-and-diagnostic-testing-strategyon-admission-to-hospital

\section{Hosted file}

Box 1.docx available at https://authorea.com/users/332647/articles/488112-covid-19-clinicalrisk-assessment-for-guiding-patient-placement-and-diagnostic-testing-strategy-onadmission-to-hospital

The intended benefits of the system were to guide patient placement and identify patients for whom a single negative PCR test might be insufficient grounds to exclude COVID-19. The latter is particularly important due to the limited sensitivity of rt-PCR on material obtained from the upper respiratory tract, , $, 4,5,6$ and the high risk to healthcare workers associated with routine deep respiratory sampling through bronchoalveolar lavage.

In preparation for an increase in COVID-19 admissions, Cambridge University Hospitals NHS Foundation Trust (CUH) set up an admissions unit run by acute physicians for those with suspected COVID-19, which was separated from the main emergency department (ED) and opened on 17th March 2020. All patients with symptoms compatible with possible COVID-19 at initial community or hospital triage were directed to the COVID-19 admissions unit. However, patients requiring higher dependency care in an ED resuscitation area (NEWS score [?]7) were deemed unsuitable.

CUH began using the COVID-19 traffic lights system trust wide in mid-March 2020. An audit of its performance in predicting cases of laboratory confirmed COVID-19 in the COVID-19 admissions unit was undertaken retrospectively via manual review of patient notes. The UK was put into lockdown with strict social distancing measures from $23^{\text {rd }}$ March 2020. Locally, the peak number of COVID-19 admissions occurred in the week beginning $8^{\text {th }}$ April 2020. From $21^{\text {st }}$ March 2020 to $14^{\text {th }}$ April 2020, of 165 audited patients to have been assigned traffic light colours by consultant physicians in acute medicine, 33 of $62(53 \%)$ labelled 'red' (high risk) were found to be SARS-CoV-2 PCR positive, while 12 of 62 (19\%) labelled 'amber' (moderate risk) and only one of $41(2 \%)$ labelled 'green' (low risk) were SARS-CoV-2 PCR positive. In the context of falling community SARS-CoV-2 transmission as a result of the UK lockdown, ${ }^{7} 5$ of $22(23 \%)$ audited patients labelled 'red' between $28^{\text {th }}$ April 2020 and $20^{\text {th }}$ May 2020 were found to be SARS-CoV-2 PCR positive, whilst 1 of $65(2 \%)$ labelled 'amber' and none of 32 'green' tested positive. Although the limited clinical sensitivity of rt-PCR on upper respiratory tract samples makes under-representation of the true rate of COVID-19 in each traffic light group likely, the proportion of 'red' patients that tested negative is notably high. This has important implications for patient placement and onward testing strategy. 
In hospitals with limited access to single-patient isolation facilities, cohort nursing of patients awaiting test results is inevitable. The traffic lights clinical risk stratification aid has proven useful in identifying non-COVID-19 patients and can enable NHS Trusts to optimise their use of available side rooms. The data above show that diagnosing COVID-19 through clinical means alone is challenging. At CUH we are currently nursing all 'red' and 'amber' patients awaiting swab results in side rooms or maximally spaced shared bays (two patients per 6-bedded bay). This strategy aims to minimise the risk of in-hospital exposures between infectious and susceptible patients. Patients badged as 'green' are routinely cohort nursed in standard shared facilities to reduce demand on available single occupancy rooms.

The data generated have also allowed us to predict the performance of additional testing strategies for excluding COVID-19, by applying published data on the sensitivity and specificity of both rt-PCR on upper respiratory tract samples and $\mathrm{CT}$ imaging of the thorax (Table 3) to patient groups in each of the traffic lights risk categories. We estimated that routine use of CT scans would likely result in overcalling COVID19 because of low specificity, even in cases of high risk 'red' patients. In view of this, we recommended repeat rt-PCR on serial upper, or preferably lower, respiratory tract samples as the most reliable way of investigating cases of ongoing diagnostic uncertainty.

\section{Hosted file}

Table 3.docx available at https://authorea.com/users/332647/articles/488112-covid-19clinical-risk-assessment-for-guiding-patient-placement-and-diagnostic-testing-strategyon-admission-to-hospital

As the pandemic unfolds, the background prevalence of COVID-19 will continue to change, and so will the proportion of patients in each traffic light risk category testing positive. We have found that the use of a risk stratification system for grading the clinical likelihood of COVID-19 at the point of initial assessment provides a helpful framework upon which to hang difficult patient placement decisions at the hospital front door, and to guide the hospital-wide response to the evolving pandemic. However, clinical diagnosis of COVID-19 has proven challenging and risks of overcalling COVID-19 should be recognised.

\section{References}

1. Collier D, Assennato S, Warne B et al. Point of Care Nucleic Acid Testing for SARS-CoV-2 in Hospitalized Patients: A Clinical Validation Trial and Implementation Study. Cell Reports Med. 2020;

2. Patterson B, Marks M, Martinez-Garcia G et al. A Novel Cohorting and Isolation Strategy for Suspected COVID-19 Cases during a Pandemic. J Hosp Infect. 2020;

3. Fang Y, Zhang H, Xie J et al. Sensitivity of Chest CT for COVID-19: Comparison to RT-PCR. Radiology. 2020;

4. Ai T, Yang Z, Hou H et al. Correlation of Chest CT and RT-PCR Testing in Coronavirus Disease 2019 (COVID-19) in China: A Report of 1014 Cases. Radiology. 2020;

5. Wong HYF, Lam HYS, Fong AH-T et al. Frequency and Distribution of Chest Radiographic Findings in COVID-19 Positive Patients. Radiology. 2019;

6. Wang W, Xu Y, Gao R et al. Detection of SARS-CoV-2 in Different Types of Clinical Specimens. JAMA - Journal of the American Medical Association. 2020;

7. Public Health England. Coronavirus (COVID-19) in the UK. Available at https://coronavirus.data.gov.uk/?_ga=2.203524627.2047977285.1592859198-753038366.1583154480

8. Guan W, Ni Z, Hu Y et al. Clinical Characteristics of Coronavirus Disease 2019 in China. N Engl J Med. 2020;

9. Shi H, Han X, Jiang $\mathrm{N}$ et al. Radiological findings from 81 patients with COVID-19 pneumonia in Wuhan, China: a descriptive study. Lancet Infect Dis. 2020;

10. Liu K, Fang YY, Deng Y et al. Clinical characteristics of novel coronavirus cases in tertiary hospitals 
in Hubei Province. Chin Med J (Engl). 2020;

11. Yang $\mathrm{X}, \mathrm{Yu} \mathrm{Y}, \mathrm{Xu} \mathrm{J}$ et al. Clinical course and outcomes of critically ill patients with SARS-CoV-2 pneumonia in Wuhan, China: a single-centered, retrospective, observational study. Lancet Respir Med. 2020 ;

12. Su L, Ma X, Yu H et al. The different clinical characteristics of corona virus disease cases between children and their families in China - the character of children with COVID-19. Emerg Microbes Infect. 2020 ;

13. Wen Z, Chi Y, Zhang L et al. Coronavirus Disease 2019: Initial Detection on Chest CT in a Retrospective Multicenter Study of 103 Chinese Subjects. Radiol Cardiothorac Imaging. 2020;2(2).

14. Bai HX, Hsieh B, Xiong Z et al. Performance of radiologists in differentiating COVID-19 from viral pneumonia on chest CT. Radiology. 2020;

15. Bernheim A, Mei X, Huang $M$ et al. Chest CT Findings in Coronavirus Disease-19 (COVID-19): Relationship to Duration of Infection. Radiology. 2020;

16. Ling Z, Xu X, Gan Q et al. Asymptomatic SARS-CoV-2 infected patients with persistent negative CT findings. European Journal of Radiology. 2020; 